\title{
MANERAS ALTERNATIVAS DE EMPODERAR JUVENTUDES. LA EXPERIENCIA DE LOS JÓVENES EN LOS MERCADOS COOPERATIVOS DEL MUNICIPIO DE PLAZA DE LA REVOLUCIÓN
}

\author{
ALTERNATIVE WAYS TO EMPOWER YOUTH. THE EXPERIENCE OF YOUNG \\ PEOPLE IN THE COOPERATIVE MARKETS OF THE MUNICIPALITY OF PLAZA \\ DE LA REVOLUCIÓN
}

YANSA DE LA CARIDAD TERRY ARAUJO

Universidad de La Habana, Departamento de Sociología. ycterry@ffh.uh.cu

RESUMEN

La consecución del llamado Cooperativismo № Agropecuario, tras la llegada de los Lineamientos de la Política Económica y Social del Partido y la Revolución, representa para Cuba la transformación teórico-práctica del cooperativismo agrícola tradicional. En primera instancia implica la expansión cooperativa a otros sectores de la economía del país. Además, conlleva a la diversificación del cooperativismo agrícola tradicional, materializado de manera específica a través de la creación de las Cooperativas Comercializadoras de Productos Agrícolas.

El presente artículo, en su pretensión primigenia de enfocar esta nueva modalidad de cooperativa en tanto forma de empleo, identifica una opción dentro del mercado de trabajo cubano actual que ha propuesto una inserción e inclusión laborales diferentes para los jóvenes. Quienes logran encontrar en estas cooperativas, si bien no un espacio que por su dinámica interna permita la continuidad de estudios, sí es una alternativa de empleo que canaliza en ellos, niveles notables de madurez, responsabilidad, compromiso, participación en la toma de decisiones y sentido de pertenencia. Asimismo, el protagonismo gradual que alcanza este segmento poblacional movido por una cultura del trabajo de nuevo tipo, le convierte en una fuerza de trabajo no subvalorada, no marginada.

PALABRAS CLAVE: Cooperativas no agropecuarias, cooperativas comercializadoras de productos agrícolas, modelo de Gestión Cooperativo, Inserción Laboral Juvenil.

\section{ABSTRACT}

The achievement of the so-called Non-Agricultural Cooperativism, after the arrival of the Guidelines for the Economic and Social Policy of the Party and the Revolution, represents for Cuba the theoretical-practical transformation of traditional agricultural cooperativism. In the first instance, it implies the cooperative expansion to other sectors of the country's economy, but it also implies the diversification of the traditional agricultural cooperativism, materialized, specifically, through the creation of the Cooperatives Commercializing Agricultural Products.

This article, in its original pretension, focus this new form of cooperative as a form of employment, identifies an option within the current Cuban labor market that has proposed a different labor insertion and inclusion for young people. Those who manage to find in these cooperatives, although not a space that due to its internal dynamics allows the continuity of studies, yes an alternative of employment that channels in them remarkable levels of maturity, responsibility, commitment, participation in the decision making and sense of belonging Likewise, the gradual protagonism reached by this segment of the population, moved by a new type of work culture, makes it a work force that is not undervalued, not marginalized.

KEYWORDS: Non-agricultural, cooperatives, cooperatives commercializing agricultural products, cooperative management model, youth labor insertion 
INTRODUCCIÓN

Tras la crisis internacional de 2008, la inserción de los jóvenes en el mercado laboral representa uno de los mayores retos que afronta el siglo XXI. La antesala de este contexto se sitúa en las manifestaciones callejeras, que emprende en marzo y abril de 2006, la juventud francesa en un escenario de constricción del mercado de trabajo europeo (ver Figura 1); marcado además, por una tasa de desempleo juvenil de un $20 \%$ (Charlin \& Weller, 2006).

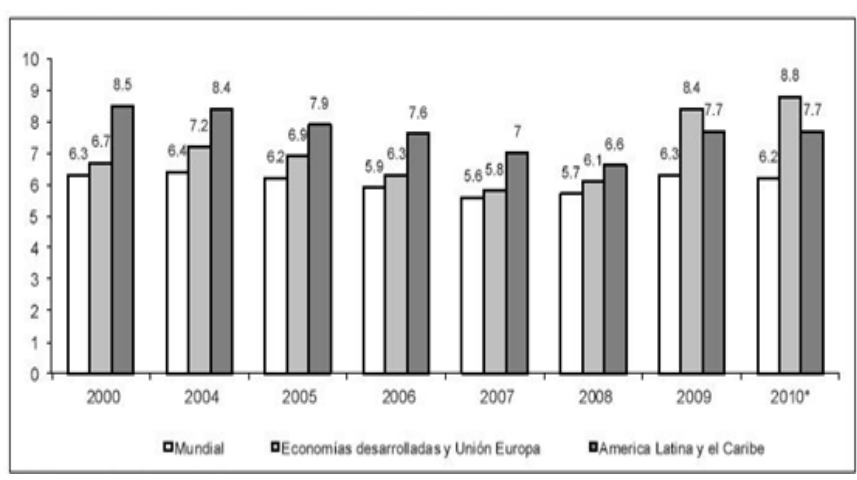

Figura 1: Tasa de desempleo mundial y por regiones Fuente: Tomado de Global Employment Trends (2011).

En Latinoamérica, la situación es muy similar. Una característica de los mercados de trabajo latinoamericanos es la persistencia de graves problemas de inserción laboral de los y las jóvenes, sobre todo, la alta precariedad en el empleo juvenil y las elevadas tasas de desempleo (Weller, 2003). Esta situación alcanza un punto álgido durante los años noventa y a inicios del presente siglo, a raíz del empeoramiento de los mercados de trabajo (Charlin \& Weller, 2006).

A nivel regional, el desempleo juvenil llega incluso a duplicar (y triplicar) la tasa de desempleo general (ver Figura 2). En 1999, la tasa de desempleo urbano general subió a $10.2 \%$, con un rango entre $2.8 \%$ en Guatemala y $19.2 \%$ en $\mathrm{Co}^{-}$ lombia. Lo que, para los jóvenes de 15 a 24 años, esta tasa se tradujo en $4.8 \%$ (Guatemala) y $36.6 \%$ (Colombia); con un promedio simple de $19.5 \%$, superándose la tasa general entre alrededor de $50 \%$ en Nicaragua y alrededor de 130 a $140 \%$ en Costa Rica, México y Uruguay (Weller, 2003).

En este marco, la no inserción de los jóvenes se convierte en un problema social de gran repercusión, por el impacto que genera en la aprehensión de estilos de vida y en procesos de constitución de las identidades de este grupo etario. Las dificultades en el acceso juvenil al mercado laboral sugieren la atrofia y subvaloración del capital humano creado para el desarrollo integral de la sociedad. Ello implica otro peligro mayor, la posible migración de la cohorte en que descansa el reemplazo poblacional y se sostiene la población económicamente activa.

El Estado cubano en su intento por crear espacios para la integración e inserción laboral juvenil- en una Cuba que no está exenta al debate ante el éxodo de jóvenes y la paradójica existencia del envejecimiento poblacional-, implementa un conjunto de políticas de contención. Estas se desarrollan en un contexto de reordenamiento laboral y reducción de plantillas infladas en el sector estatal de la economía, a través de los Lineamientos de la Política Económica y Social del Partido y la Revolución, aprobados el 18 de abril de 2011 en el VI Congreso del PCC.

En 2013 el llamado Cooperativismo No Agrícola (García, Nova y Anaya, 2014) constituye una de las propuestas para promover la generación de empleo estable y no precario, eliminar las trabas burocráticas (en aspectos como la contratación laboral) y potenciar niveles de descentralización, autonomía, autogestión y participación (Nova, 2012). Sin lugar a dudas, desde aquí se propicia un pie forzado para la expansión del cooperativismo (agrícola) tradicional a otros sectores de la economía, que deviene

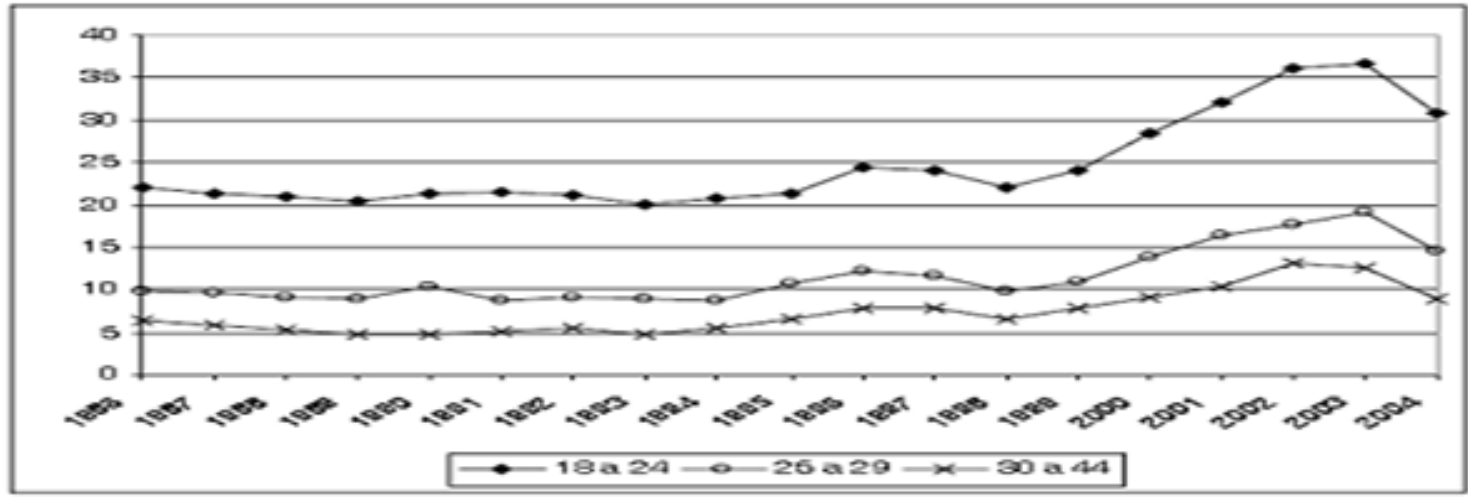

Figura 2. Tasa de desempleo para grupos de edad (en \%) Fuente: Tomado de Bucheli (2006) 
mayor visibilidad en el espacio urbano de la fórmula cooperativa y su legitimación en tanto opción de empleo alternativa al modelo estatal pero funcional a los principios del socialismo en Cuba.

Sin embargo, el Cooperativismo No Agrícola también apertura la diversificación de la experiencia cooperativa tradicional mediante la implementación de Cooperativas Comercializadoras de Productos Agrícolas (en lo adelante CCPA o Mercados Cooperativos) (ver Figura 3).

En una esfera estratégica y desfavorecida ${ }^{1}$, esta forma de comercialización representa un intento por reorganizar el sistema de comercialización agrícola, estrechar vínculos entre producción y consumo, frenar la baja eficiencia en el sector y el predominio de la oferta, estimular la producción y satisfacer la demanda de alimentos de la población (Nova, 2012).
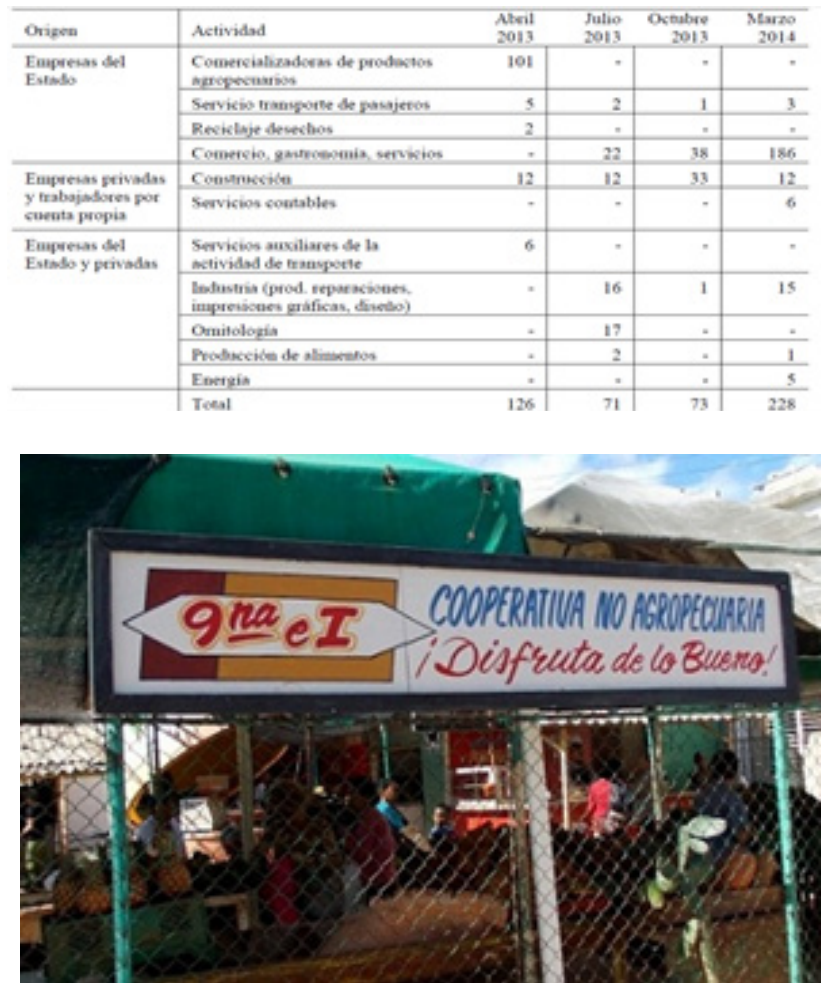

Figura 3. Cooperativas no agropecuarias aprobadas por el Consejo de Ministros $2013-2014$

Fuente: Tomado de Vuotto y Verbeke (2014)

1. La comercialización de productos agrícolas a la llegada de los Lineamientos se caracterizaba, bajo el monopolio estatal por: la baja productividad en el sector, el dominio de la oferta versus la demanda, la pérdida de cosechas, atraso en el pago a productores, el desestímulo de los productores, entre otros elementos (Bu y Rego, 2007) (Nova, 2010).
De ahí, su implementación por decisión estatal, como parte del paquete de medidas desarrolladas entre 2007-2014 para dinamizar el sector; dando inicio al proceso de cooperativización del Mercado Agropecuario Estatal (MAE), y paso a otra forma de empleo en el sector.

A grosso modo, el Mercado Cooperativo apertura una concepción auto-subsidiada y autónoma en el mercado agropecuario, que subvierte el paternalismo y asistencialismo del Estado en el $\mathrm{MAE}^{2}$. Se distingue dentro de la atomizada escena de la comercialización agrícola ${ }^{3}$, por la venta de algunos productos a precios de acopio centralizado a minoristas máximos (en el caso de la papa, el arroz, el chícharo, el huevo y la carne de cerdo) y el resto a precios de oferta y demanda (Vuotto, 2015) (ONEI, 2015). Pero, ¿qué implicancias tiene para la juventud? ¿Se convierte en una opción de empleo interesante para los jóvenes?

En un intento por explicar las interrogantes planteadas, el presente artículo se apoya en una investigación realizada por la autora, que asume una perspectiva sociológica y la articulación entre un enfoque contextual (de la cooperativa en su entorno) y un enfoque funcional (de la cooperativa para sí) (Bu y Rego, 2007). Se trata de un eje de análisis movido por la sensibilidad del tema en el contexto cubano actual, y la escasa investigación producida en materia de comercialización agrícola desde un análisis crítico y con enfoque sistémico e integral.

\section{METODOLOGÍA}

La investigación que da cabida al presente artículo constituyó un estudio de casos múltiples en un contexto específico, según se tomaron como unidades de estudio el universo de los Mercados

2 Bajo el modelo cooperativo, los trabajadores de manera colectiva asumen todos los gastos (y pérdidas) que demanda la operación comercial y que generan como cooperativa (el pago de los servicios de telefonía, gas o electricidad).

3 Desde la Gaceta Oficial Extraordinaria № 35/2013 se establecen de manera experimental en las provincias de La Habana, Artemisa y Mayabeque nuevas fórmulas de comercialización de productos agropecuarios que conllevan a la expansión de la red de mercados minoristas (mercados agropecuarios con administración y gestión estatal, mercados gestionados por cooperativas no agropecuarias, mercados arrendados y mercados de oferta y demanda). 
Cooperativos del municipio de Plaza de la Revolución (CCPA 26 y 41, CCPA 25 y 10, CCPA 9na e I y CCPA Loma y Tulipán). Para levantar el estado de opinión de los jóvenes cooperativistas se utilizó la técnica de la entrevista semi-estructurada, con vistas a agilizar la comunicación. Se aplicaron un total de 10 entrevistas, que representan el $80 \%$ de los jóvenes de entre 23-30 años que trabajan en los Mercados Cooperativos seleccionados como unidades de estudio.

El municipio Plaza de la Revolución es seleccionado de manera intencional porque, por una parte, constituye un espacio económico próspero dado que cuenta con uno de los mayores niveles en cuanto a densidad poblacional, salarios, volumen de inversiones o ingresos (ONEI, 2015). Por otra, es uno de los municipios no sólo más densamente poblados sino más envejecidos, y en consonancia más necesitados de la funcionalidad de mecanismos de comercialización más justos y solidarios, y de formas empresariales socialmente responsables. De la misma manera que se convierte la escasa cultura del trabajo agrícola en el municipio (por su ubicación geográfica) en un elemento interesante a ser considerado, para polemizar sobre el nivel de acogida de esta opción de empleo por las poblaciones más jóvenes.

Metodológicamente, se aplicó un análisis integral que imbrica una perspectiva funcional y otra contextual ${ }^{4}$. Eso según la articulación de una dimensión socio-cultural en un determinado marco de relaciones económico-contractuales, conecta la cuestión de los valores y pautas culturales con procesos como la contratación laboral o la composición de la estructura organizativa, en un marco normativo socialmente determinado.

DESARROLLO

La participación de los jóvenes en las Cooperativas Comercializadoras estudiadas está dada

4 El enfoque funcional considera la dinámica que adoptan las relaciones sociales en el proceso de intercambio de bienes y servicios, tomando en cuenta el medio ambiente socio-laboral, así como determinadas relaciones económicas y contractuales. El enfoque contextual contempla los elementos externos a las cooperativas que pueden incidir (o inciden) en su gestión como las condiciones climáticas, el marco normativo, las exigencias sociales o el papel del Estado ( $\mathrm{Bu}$ y Rego, 2007). fundamentalmente a partir de un proceso de contratación inicial de personal. En el 75\% de las cooperativas del municipio de Plaza se efectúa este proceso de contratación, a raíz de la baja de personal directivo y no directivo, ya fuere por motivos económicos o por actos de indisciplina laboral.

Las barreras que presentan los Mercados Cooperativos $^{5}$ y la permanencia de gran parte de la fuerza de trabajo inicial (calificada para el trabajo por sus años de experiencia y trayectoria laboral en el sector) son factores centrales que sustentan la reducción de la contratación y el énfasis en la idoneidad del trabajador. La contratación limitada de personal parte además, de la asunción de una política hacia la contratación de fuerza de trabajo asalariada:
"tenemos contratos de servicios de trabajo a cuentapropistas. Hemos tenido que contra- tar personal para estibar, para la limpieza... Pero no son socios. Ahora no estamos con- tratando personal para trabajar aquí porque tenemos que funcionar con menos personal porque esto va a determinar nuestro nivel de ganancias." (Fragmento de entrevista reali- zada en CCPA de 26 y 41).

Con base en los elementos antes expuestos, se realiza una contratación de personal limitada en las cooperativas estudiadas, que conlleva a la contracción del número de trabajadores, la selección o exclusión de determinados grupos poblacionales a partir de sus condiciones y potencialidades. No obstante, los jóvenes encuentran en este contexto un espacio de inserción, como segmento etario con mayores posibilidades de cubrir las expectativas de la Asamblea General de todos los socios en el proceso de contratación, de aptitud para el trabajo y nivel de preparación.

En el Mercado Cooperativo, el joven constituye una fuerza laboral idónea por sus capacidades y habilidades para el trabajo. Tal es así que se propicia, incluso, la contratación de jóvenes desvinculados del estudio y sin una experiencia laboral previa. Característica que marca una ruptura importante con la lógica interna en algunos espacios dentro del sector no estatal,

5 En una investigación realizada por la autora (Terry, 2016) se identificaron una serie de barreras políticas, culturales, educativas, logístico-materiales, institucionales y en la gestión interna. 
y en determinadas modalidades cooperativas, donde tiende a complejizarse el conjunto de exigencias para la contratación de personal (presentación personal, nivel educacional, experiencia laboral en el área de trabajo o dominio de idiomas extranjeros).

A su vez, el sistema de retribución en las cooperativas mediante el pago mensual de un anticipo de las utilidades y el pago anual de las utilidades, se convierte en el principal atractivo para la inserción de los jóvenes en estas cooperativas. Lo que hace de esta forma de comercialización una vía para materializar la propuesta del Lineamiento 198: "priorizar la adopción de medidas para incentivar la incorporación y permanencia de jóvenes al sector agropecuario" (PCC, 2011). En este sentido, la posibilidad de mayores ingresos desde la aplicación de la lógica de distribución de "a cada cual según su trabajo", implica no sólo un incentivo para el joven trabajador sino también la reducción de las brechas salariales entre adultos y jóvenes, en contraposición a las tendencias que asume el mercado de trabajo en la región (Weller, 2003).

En el municipio de Plaza, las CCPA de Loma y Tulipán y la de 26 y 41 representan la máxima expresión de la inserción de los jóvenes a este nuevo modelo de gestión. En ambas cooperativas, alrededor de un $40 \%$ del total de socios tienen menos de 30 años de edad, motivados no sólo por la posibilidad de mayores ingresos, sino también por el apoyo de la familia que "prefiere que esté trabajando aquí a que esté robando en la calle" (Fragmento de entrevista realizada en la CCPA de Loma y Tulipán).

La familia se convierte en uno de los elementos clave que potencia la inserción de estos jóvenes en dichos espacios. Representa para estas familias, una manera de crear trabajo decente, no precario, productivo y lucrativo en jóvenes desvinculados del estudio, faltos de experiencia laboral, o desocupados.

En un análisis de los jóvenes que componen la estructura organizativa en estas cooperativas es posible hablar de la masculinización de la fuerza de trabajo. En su mayoría con un color de piel blanca o mestiza, cuyo nivel educacional se mueve entre el nivel medio y superior (un $70 \%$ tiene 12 grado y el $30 \%$ posee nivel de técnico medio y universitario). Un $50 \%$ de la fuerza de trabajo juvenil se insertan a las cooperativas por medio de terceros (familiares o amigos) y reside cerca de las cooperativas en las que trabajan, en contra de los estereotipos que circulan en las localidades urbanas.
Los hombres jóvenes que componen la estructura organizativa en los mercados cooperativos estudiados, en su mayoría están casados o unidos, constituyen una forma de exteriorizar así sus responsabilidades en el ámbito doméstico y garantizar su reproducción como ser humano ${ }^{6}$. En este sentido, muchos de ellos comentan "cuando uno llega a la casa está mata' o, y sólo quiere comer y dormir" (Fragmento de entrevista realizada en la CCPA de 25 y 10). En el caso de las féminas jóvenes, éstas utilizan el apoyo familiar (básicamente de otras mujeres), como recurso para poder desempeñar sus funciones en la vida laboral y lidiar con sus roles tradicionales en el hogar. Un hecho que manifiesta el traspaso a la mujer de las responsabilidades respecto al cuidado familiar; lo que muestra la presencia de tipos de relaciones que consolidan una cultura patriarcal.

Desde un enfoque de género, las mujeres jóvenes que componen estos espacios cuentan por lo general con ciertas concesiones: "Empecé trabajando aquí porque tenía un niño chiquito y el horario es más abierto para mí, y los muchachos me ayudan mucho. Imagínate soy la única mujer..." (Fragmento de entrevista realizada en la CCPA de Loma y Tulipán). Pero a la postre, tales concesiones determinan el rol colateral y el carácter pasivo de la mujer en este escenario; que, si bien no tiene una repercusión importante en la distribución de los ingresos, lo hace en la definición de los cargos en la estructura organizativa. Por ello, si bien las féminas logran escalar posiciones jerárquicas en las cooperativas - a partir de su nivel de conocimientos, desempeño y trayectoria laboral- el ascenso de aquellas devenidas socias, sólo ocurre en caso de que no exista una contrapartida masculina en condiciones relativamente similares.

En correspondencia con esto, los hombres jóvenes llevan a cabo procesos de movilidad ocupacional ascendente más expeditos. Un número considerable de (hombres) jóvenes ocupan en la Junta Directiva, los puestos más cercanos a la Presidencia, según su desempeño laboral en el área designada y/o alto nivel educacional.

6 Entiéndase este término como un importante aspecto de la reproducción social, que incluye el trabajo necesario para la reproducción de la vida y que ha sido realizado históricamente por la mujer (cuidados necesarios para el sostenimiento de la vida, alimentación, cuidados sanitarios, apoyo psicológico, mantenimiento de los espacios y bienes domésticos, entre otros). 
Incluso algunos de ellos, ante la baja de directivos y la posibilidad de aumentar las ganancias, han conseguido ascender a la Presidencia (como ocurre en la CCPA de 26 y 41 y en la de Loma y Tulipán).

Cabe señalar que el rejuvenecimiento de la Junta Directiva repercute de manera positiva en la gestión interna del espacio cooperativo. En aquellas unidades donde se produce una movilidad ascendente en los jóvenes hay un mayor uso de los principios cooperativos y un mejor aprovechamiento del margen de posibilidades que brinda el marco regulatorio. Debido a esto, existe un consenso entre los trabajadores con más experiencia y años de trabajo de que: "Con la entrada de estos muchachitos jóvenes se ha dinamizado mucho el trabajo en el mercado..." (Fragmento de entrevista realizada en la CCPA de Loma y Tulipán).

De igual forma, y en contradicción con los imaginarios sociales que hablan de una juventud perdida, el ascenso del joven cooperativista hasta los escaños más elevados de la estructura organizativa ha compulsado a la participación en la toma de decisiones y propiciado el compromiso, la responsabilidad y el sentido de pertenencia en este segmento poblacional.

En base a lo anterior, vale decir que la experiencia del Mercado Cooperativo ha obrado a favor de la socialización del joven en una cultura del trabajo de nuevo tipo, en la generación de valores y en la expansión de los beneficios más allá de lo económico; todo ello, amén de las irregularidades y dificultades que muestra la CCPA, marcado por un conjunto de barreras fundamentalmente estructurales (logístico-materiales, educativo- informativas, institucionales, político-normativas e ideológico-culturales). Como respuesta, se ha hecho manifiesta la resistencia e incondicionalidad de muchos de estos jóvenes, quienes en tales condiciones han sido un canalizador importante de acciones estratégicas al interior de las unidades de estudio (Terry, 2016). De ahí, cabría preguntarse, ¿La juventud está perdida o sólo precisa de los mecanismos para encontrarse?

\section{CONSIDERACIONES FINALES}

Las Cooperativas Comercializadoras de Productos Agrícolas, con todos sus tropiezos, son otra de las oportunidades de mitigar las vulnerabilidades de los jóvenes marginados del mercado de trabajo cubano y de incrementar los más diversos capitales para este grupo en el contexto de crisis actual. Porque sin dudas, tiene de positivo el haber otorgado a los jóvenes (y no necesariamente más favorecidos de la capital), beneficios en más de un sentido, que llegan aparejados de una cultura del trabajo de nuevo tipo frente al tradicional modelo estatal.

No obstante, lo que podría representar para los jóvenes una alternativa transgresora y sui géneris entre las opciones de empleo del mercado laboral cubano, pareciera poco sostenible, lastrado por las barreras antes mencionadas. En este sentido, y con la intencionalidad de romper barreras se plantean una serie de recomendaciones:

- Implementar cursos introductorios a todos los asociados, sin distinciones entre sujetos con cargos directivos y no directivos.

- Crear instituciones que supervisen, representen y apoyen el desarrollo de las Cooperativas No Agropecuarias.

- Generar condiciones materiales mínimas y que se desarrolle un mercado de insumos para la organización y gestión cooperativa.

- Que se promueva una mayor articulación entre gobiernos locales y cooperativas.

- Que se habiliten las cooperativas de segundo grado.

- Que exista un proceso de análisis y supervisión en toda la cadena de producción, distribución y comercialización.

- Que se implementen políticas dirigidas a la comercialización sobre la base de un enfoque sistémico-integral.

- Que se haga énfasis en el papel social de las cooperativas por parte de las instituciones supervisoras y en cursos introductorios a los cooperativistas.

- Proporcionar a los cooperativistas cursos de cooperativización, gestión empresarial, comercialización y otros, definidos a partir de un estudio de necesidades de aprendizaje; así como que se habiliten oficinas especializadas en asesoría en comercialización y formación de empresas dirigidas a las formas no estatales. 


\section{REFERENCIAS BIBLIOGRÁFICAS}

$\mathrm{Bu}, \mathrm{A}$. y Rego, I. (2007). Cuba: producción, transformación y comercialización de productos agropecuarios. Agroalimentaria, 13 (25), 13-32.

Bucheli, M. (2006). Mercado de trabajo juvenil: situación y políticas. Montevideo, Uruguay: Oficina de la CEPAL.

Charlin, M. y Weller, J. (2006). Juventudy Mercado Laboral:Brechasy Barreras. Santiago, Chile Presentación: FLACSO-Chile, CEPAL.

García, A., Nova, A., y Anaya, B. (2014). Despegue del sector agropecuario: Condición necesaria para el desarrollo de la Economía Cubana. En Colectivo de autores, Economía Cubana: Transformaciones y Desafíos (pp. 197-260). La Habana, Cuba: Editorial de Ciencias Sociales.

Global Employment Trends. (2011). Tasa de desempleo mundial y por regiones. Ginebra: Global Employment Trends.

Nova, A. (2012). Teoría y práctica en los Lineamientos de la Política Económica y Social. Revista Temas, 72, 78-82.

Nova, A. (2010). La cadena comercializadora en el sector agropecuario cubano. Recuperado de www.espaciolaical.org/contens/22/8184.pdf.
ONEI. (2015). Anuario Estadístico 2014. Recuperado de http://www.onei.cu

Terry, Y. (2016). Relaciones socioeconómicas entre los Mercados Cooperativos del municipio de Plaza de la Revolución. Universidad de La Habana, Facultad de Filosofía e Historia. Departamento de Sociología. Tesis de Diploma. La Habana.

Partido Comunista de Cuba. (2011). Lineamientos de la Política Económica y Social del Partido y la Revolución. Recuperado de https://www.pcc. cu/sites/default/files/documento/pdf/20180426/ lineamientos-politica-partido-cuba.pdf

Vuotto, M. (2015). Las Cooperativas No Agropecuarias y la transformación económica en Cuba: políticas, procesos y estrategias. REVESCO. Revista de Estudios Cooperativos (120), 149-181.

Vuotto, M., y Verbeke, G. (2014). Las nuevas formas institucionales del cooperativismo cubano: modelos estrategias y desafíos. XII Congreso Nacional de Estudios del Trabajo. Buenos Aires, Agosto 2014.

Weller, J. (2003). La problemática inserción laboral de los y las jóvenes. Santiago de Chile, Chile: Naciones Unidas. 